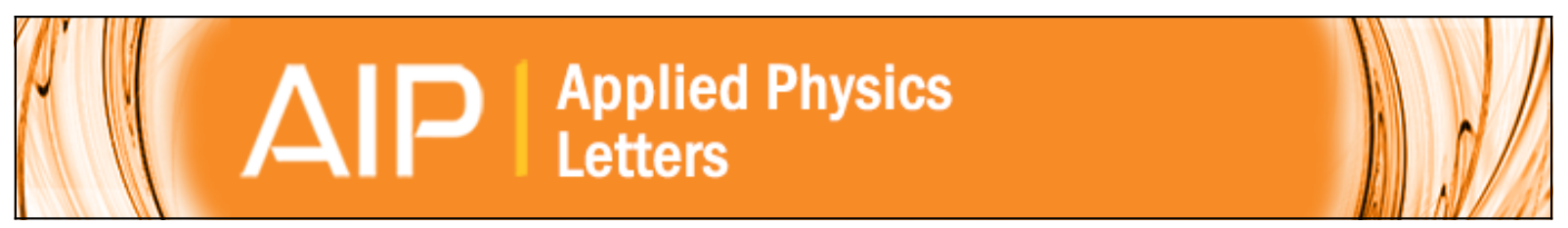

\title{
Current-controllable planar S-(S/F)-S Josephson junction
}

O. Vávra, W. Pfaff, R. Monaco, M. Aprili, and C. Strunk

Citation: Applied Physics Letters 102, 072602 (2013); doi: 10.1063/1.4792213

View online: http://dx.doi.org/10.1063/1.4792213

View Table of Contents: http://scitation.aip.org/content/aip/journal/apl/102/7?ver=pdfcov

Published by the AIP Publishing

\section{Articles you may be interested in}

Non-equilibrium photoexcited carrier effects in a graphene-based Josephson junction

Appl. Phys. Lett. 108, 033109 (2016); 10.1063/1.4940377

Supercurrent in $\mathrm{Nb} / \mathrm{InAs}$-nanowire/Nb Josephson junctions

J. Appl. Phys. 112, 034316 (2012); 10.1063/1.4745024

Planar S-(S/F)-S Josephson junctions induced by the inverse proximity effect

Appl. Phys. Lett. 95, 062501 (2009); 10.1063/1.3200226

Planar Mg B 2 superconductor-normal metal-superconductor Josephson junctions fabricated using epitaxial Mg B $2 /$ Ti B 2 bilayers

Appl. Phys. Lett. 88, 222511 (2006); 10.1063/1.2208555

Josephson junctions with nearly superconducting metal silicide barriers

Appl. Phys. Lett. 87, 222511 (2005); 10.1063/1.2137992

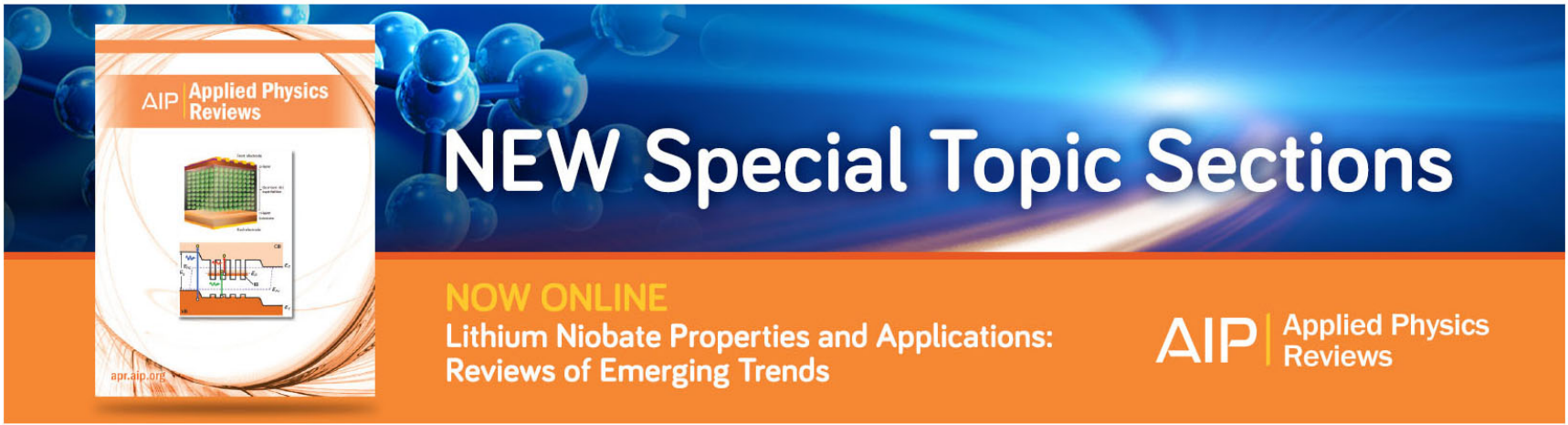




\title{
Current-controllable planar S-(S/F)-S Josephson junction
}

\author{
O. Vávra, ${ }^{1,2, a)}$ W. Pfaff, ${ }^{1}$ R. Monaco, ${ }^{3}$ M. Aprili, ${ }^{4}$ and C. Strunk ${ }^{1}$ \\ ${ }^{1}$ Institute for Experimental and Applied Physics, University of Regensburg, D-93040 Regensburg, Germany \\ ${ }^{2}$ Nanoelektronik, Technische Fakultät, Christian-Albrechts-Universität zu Kiel, 24143 Kiel, Germany \\ ${ }^{3}$ Istituto di Cibernetica del CNR, Comprensorio Olivetti, I-80078 Pozzuoli, Italy \\ ${ }^{4}$ Laboratoire de Physique des Solides, Université Paris-Sud, CNRS, UMR 8502, 91405 Orsay Cedex, France
}

(Received 12 December 2012; accepted 31 January 2013; published online 20 February 2013)

We report on the experimental realization of a current-controllable lateral S-(S/F)-S Josephson junction based on the inverse proximity effect in the superconductor-ferromagnet bilayer (S/F). The dependence of the critical current on the magnetic field $\mathrm{I}_{c}(\mathrm{~B})$ shows a Fresenel-like pattern, which could qualitatively be understood with the theory of Josephson junctions in a magnetic field gradient. The amplitude and the period of the $\mathrm{I}_{c}(\mathrm{~B})$ pattern can be controlled by spin-polarized quasiparticles injection into the weak link. The period change suggests controllability of effective area of the Josephson junction. Furthermore, a temperature-induced transition from a weak-link behavior to a strong coupling between the superconducting banks is also observed in these lateral Josephson junctions. (C) 2013 American Institute of Physics. [http://dx.doi.org/10.1063/1.4792213]

The controllability of the supercurrent in a Josephson junction ${ }^{1}$ has attracted a considerable attention and can be achieved in several ways. For instance, in a weak link, where the junction is created by two superconducting banks (S) separated by a normal metal $(\mathrm{N})$, the supercurrent can be controlled by a transversal current flow through the normal region, ${ }^{3}$ in this case the observed suppression of the supercurrent is due to the presence of hot electrons in the normal metal. Moreover, by controlling the energy distribution of the current carrying states in the N-part of the weak link, one can even reverse the direction of the supercurrent. ${ }^{4,5}$ Nevertheless, there is a lack of the work showing the control over both the supercurrent and the effective area of the Josephson junction which affects its magnetic properties.

In a previous article, ${ }^{6}$ we presented the utilization of the inverse proximity effect ${ }^{7}$ in a superconductor-ferromagnet (S-F) bilayer to generate a lateral weak link of S-(S/F)-S type by the proximity-induced suppression of the superconducting order parameter. We used a bilayer cross strip geometry, i.e., an $\mathrm{F}$ strip on top of a $\mathrm{S}$ strip. The critical temperature of the superconducting layer was suppressed in the $S / F$ intersection area. Furthermore, at appropriate thicknesses of $\mathrm{S}$ and $\mathrm{F}$ layers, the structure revealed the dc and ac Josephson effect.

In this paper, we demonstrate a controllable planar S-(S/ F)-S Josephson junction that is realized by injecting quasiparticles from the ferromagnet into a superconductor. To increase spin-polarization, we used iron $(\mathrm{Fe})$ as ferromagnet; hence a weak link is formed by the inverse proximity effect in the $\mathrm{Nb} / \mathrm{Fe}$ bilayer.

The layout of the junction is shown in Fig. 1(a). The structure was defined by electron beam lithography in the following steps: (i) Au alignment markers and electrodes for injection were thermally evaporated and patterned by a positive PMMA lithographic lift-off process, (ii) a $20 \mathrm{~nm}$ tick $\mathrm{Nb}$ layer was e-gun evaporated in a ultra high vacuum chamber,

\footnotetext{
${ }^{\text {a) }}$ Author to whom correspondence should be addressed. Electronic mail: ondro.vavra@gmail.com.
}

then patterned into a $900 \mathrm{~nm}$ wide strip by negative ARN resist and etched by $\mathrm{SF}_{6}$ reactive ion etching, (iii) a $15 \mathrm{~nm}$ thick Fe layer was e-gun evaporated and lifted-off by a positive PMMA process to form a $220 \mathrm{~nm}$ wide strip orthogonal to and on top of the $\mathrm{Nb}$ layer. To ensure good electrical contact, the short rf-plasma cleaning of the Au electrodes and $\mathrm{Nb}$ strip was done right before the $\mathrm{Fe}$ deposition. At $\mathrm{T}=4.2 \mathrm{~K}$, the interfacial resistance between the $\mathrm{Nb}$ and $\mathrm{Fe}$ strips was about $10 \mathrm{~m} \Omega$, measured in the configuration denoted by red letters in Fig. 1(a). All critical current $\left(\mathrm{I}_{c}\right)$ measurements were performed using a 4-point lock-in method in the way that the differential resistance $d V / d I$ vs. the bias current I is measured. Then, the critical current was defined as the value where the onset of resistance appears (more in Figs. 2(b) and 2(c)).

The critical current $\mathrm{I}_{c}$ of the $\mathrm{Nb}-(\mathrm{Nb} / \mathrm{Fe})-\mathrm{Nb}$ weak link was measured in the configuration denoted by the light blue letters in Fig. 1(a). The temperature dependence of the critical current $\mathrm{I}_{c}$, shown in Fig. 1(b), reveals three different regimes. In the upper range from $6.4 \mathrm{~K}$ up to the critical temperature of the $\mathrm{Nb}$ film $\mathrm{T}_{c}(6.8 \mathrm{~K})$, we observed the Josephson effect. At temperatures below $6.1 \mathrm{~K}$, a regular superconducting condensate was developed and found to dominate over the weak link behavior. In between 6.1 and $6.4 \mathrm{~K}$, the two mechanisms were shown to compete.

For a better understanding of these regimes, we measured the threshold curves $I_{c}(B)$ with a magnetic induction field B applied perpendicularly to the junction plane, as indicated in Fig. 1(a). The threshold curves at various temperatures are displayed in Fig. 3(a). With decreasing temperature, the curves gradually develop into three distinctive types of behaviours. At 6.5 and $6.6 \mathrm{~K}$, within the Josephson regime, the interference patterns are well defined. In between 6.1 and $6.5 \mathrm{~K}$, the main peak is not rounded anymore but seems to be cut off on the top. Below $6.1 \mathrm{~K}$, as expected from the previous measurements, ${ }^{6}$ the $\mathrm{I}_{c}(\mathrm{~B})$ curves could not be well defined. This is due to the fully developed superconducting condensate leading to a strong coupling between the Nb banks. In other words, at sufficiently low temperatures the local suppression of the pair amplitude by the ferromagnet $(\mathrm{Fe})$ is unable to 

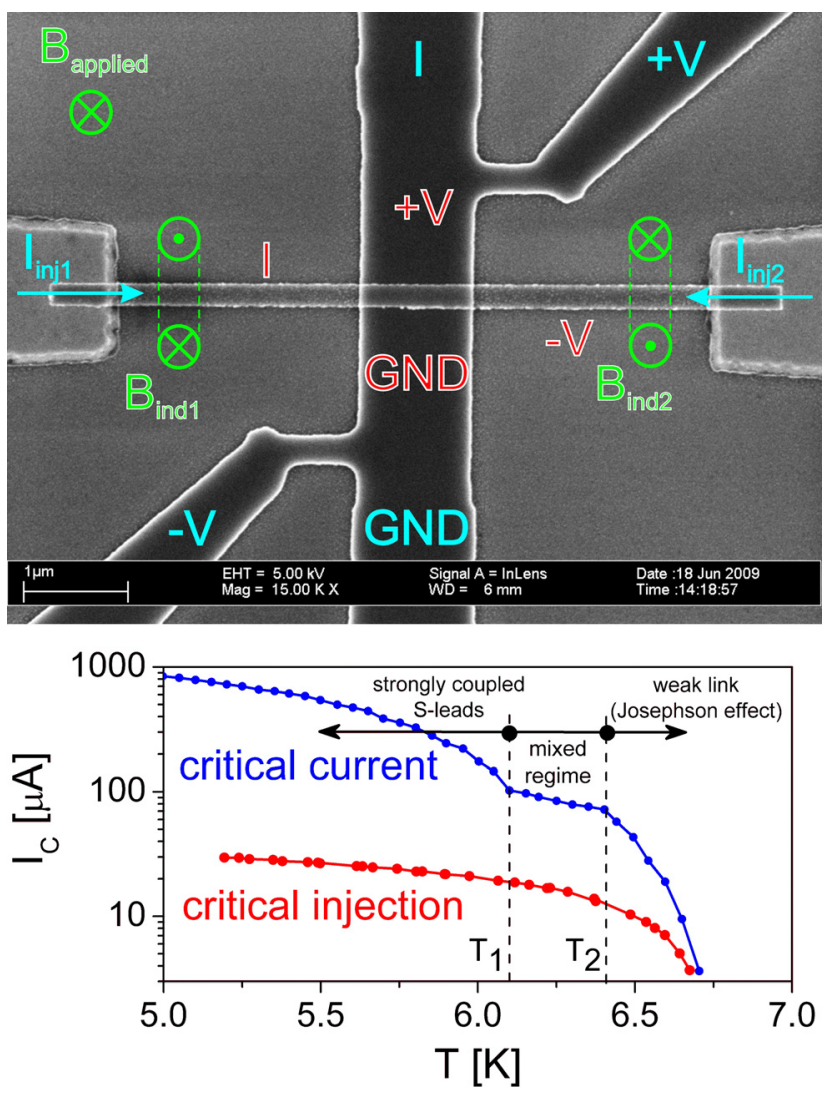

FIG. 1. (a) Sample micrograph. The Josephson weak-link is formed where the narrow horizontal $\mathrm{Fe}$ strip crosses the vertical wide $\mathrm{Nb}$ strip. The light blue labels denote the current and voltage contacts for the four-point differential resistance measurements with injection currents $\mathrm{I}_{i n j 1,2}=\mathrm{I}_{i n j} / 2$. The red labels indicate how the Nb-Fe interfacial resistance is measured. (b) Temperature dependences of the critical current $I_{c}$ and of the critical injection current $\mathrm{I}_{i n j, C}$.

form a proper weak link. The $\mathrm{I}_{c}(\mathrm{~B})$ curves at below $6.1 \mathrm{~K}$ exhibit a "roof"-like structure on top of the zero-field peak. To identify this feature, the behavior around zero magnetic field was measured in detail on different samples. The two
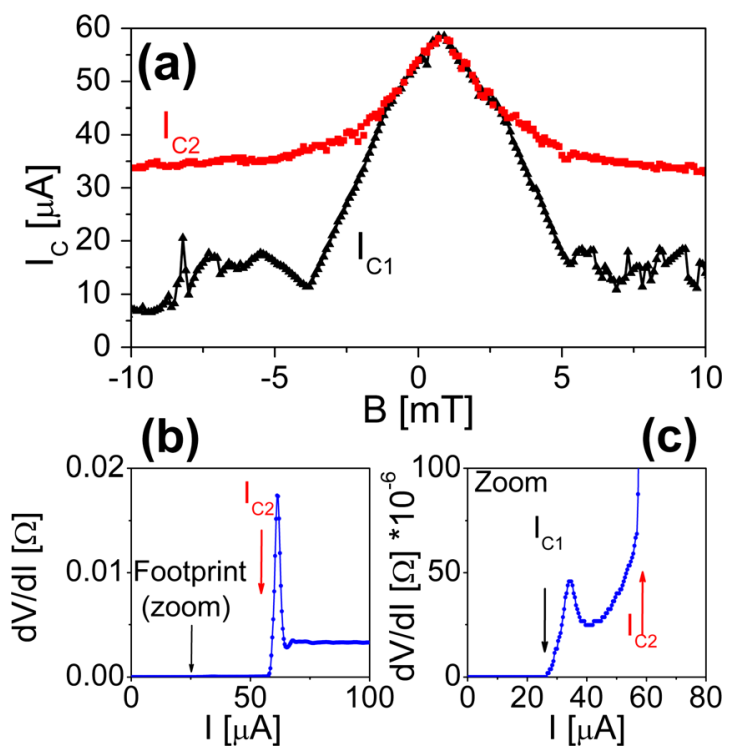

FIG. 2. (a) The $\mathrm{I}_{c}$ (B) curve explaining its "roof feature." Differential dI/dV curves with two critical current values: (b) $\mathrm{I}_{c 1}$ is taken with the lower resistance criterion and $\mathrm{I}_{c 2}$ with the higher resistance criterion. resistance criteria adopted during the $\mathrm{dV} / \mathrm{dI}$ measurement resulted in two different $\mathrm{I}_{c}$ values, and thus two different $\mathrm{I}_{c}(\mathrm{~B})$ behaviours as shown in Fig. 2(a). The first onset of the resistance (low current criterion) corresponds to the critical current, $\mathrm{I}_{c 1}$, of the weak link, whereas the second current jump, $\mathrm{I}_{c 2}$, on the differential resistance curve (high current criterion) corresponds to the transition of the $\mathrm{Nb}$ leads into the normal state. The low and high criteria are shown in Figs. 2(b) and 2(c), respectively. At a certain magnetic field [2 $\mathrm{mT}$ in Fig. 2(a)], the two curves merge into one, implying that the superconducting condensate is so strong that the influence of the inverse proximity effect can be neglected.

As shown in Fig. 1(a), the injection current, $\mathrm{I}_{i n j}$, is split into two equal currents on both sides of the $\mathrm{Fe}$ injector, $\mathrm{I}_{i n j 1}=\mathrm{I}_{i n j 2}=\mathrm{I}_{i n j} / 2$ and injected into the Nb leads; the voltage was measured between the probes labeled $\mathrm{V}+$ and $\mathrm{V}-$. The red curve in Fig. 1(b) shows the measured temperature dependence of the critical injection current, $\mathrm{I}_{i n j, c}$, defined as the minimum injection current required to drive the $\mathrm{Nb} / \mathrm{Fe}$ bilayer into the normal state.

However, the most visible impact of the injected spinpolarized quasi-particles into the $\mathrm{Nb}-(\mathrm{Nb} / \mathrm{Fe})-\mathrm{Nb}$ weak link can be seen on the interference patterns $\mathrm{I}_{c}(\mathrm{~B})$ shown in Fig. 3 (b). Both amplitude and period of the patterns decrease with increasing the injection current $\mathrm{I}_{i n j}$. Above all, the threshold curves do not represent the Fraunhofer-like patterns, which are typically measured in homogeneous Josephson junctions immersed in a uniform magnetic field.

In our case, the patterns rather mimic the Fresnel-like behavior predicted and experimentally verified for the Josephson junctions exposed to an inhomogeneous magnetic field. ${ }^{8,9}$ In our experiment, the inhomogeneity of the

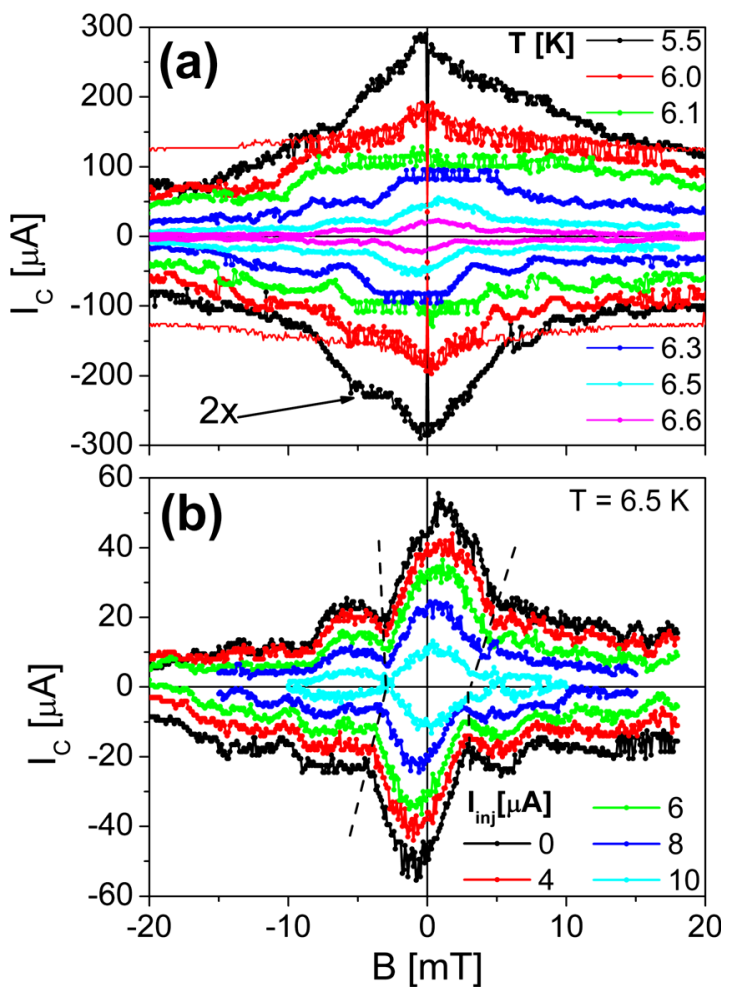

FIG. 3. (a) The temperature development of critical current vs. magnetic field pattern $\mathrm{I}_{c}(\mathrm{~B})$, (b) the dependence of the $\mathrm{I}_{c}(\mathrm{~B})$ pattern on the injection current $\mathrm{I}_{i n j}$ at $6.5 \mathrm{~K}$ (dashed guide lines indicate the change of the period). 


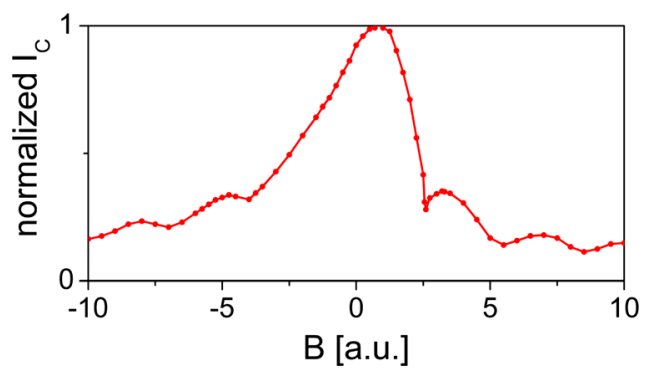

FIG. 4. Numerically computed threshold current $I_{c}(B)$ for a long overlap-type Josephson junction with uniform bias and asymmetric boundary conditions.

magnetic field comes from the shape of the injectors (e.g., the step at the $\mathrm{Nb}$ edges) and the injection currents flowing via the Fe strips into the $\mathrm{Nb}$. Since they flow in opposite direction they generate opposite magnetic fields, $B_{1}=-B_{2}$, i.e., a magnetic field gradient.

The skewness of the threshold curves in Fig. 3(b) unequivocally indicates the presence of the self-field effect. ${ }^{10}$ This effect can typically be observed in long Josephson junctions, that is, when one of the junction dimension (in our case the width, $w=900 \mathrm{~nm}$, of the $\mathrm{Nb}$ strip) is larger than the Josephson penetration depth ${ }^{1} \lambda_{J}=\sqrt{\Phi_{0} / 2 \pi \mu_{0} j_{c} d}$, where $\Phi_{0}$ is the magnetic flux quantum, $\mu_{0}$ the vacuum permeability, $j_{c}$ is the Josephson critical current density, and $d$ the weak link magnetic thickness given by the sum of Fe-strip width $(220 \mathrm{~nm})$ and the field penetration into the S-parts on each side of the weak link. For our thin Nb layer, the London penetration depth $\lambda_{L}$ has to be replaced by the larger Pearl penetration depth ${ }^{2} \lambda_{P}=\lambda_{L}^{2} / t_{N b}$ where $t_{N b}=20 \mathrm{~nm}$ is the $\mathrm{Nb}$ thickness. Assuming $\lambda_{L} \simeq 45 \mathrm{~nm}$, we obtain $\lambda_{P} \simeq 100 \mathrm{~nm}$ and $d \simeq 420 \mathrm{~nm}$. Assuming the current density to be uniform over the weak link cross section, $w \times t_{N b}$, the critical current of about $50 \mu \mathrm{A}$, measured at $6.5 \mathrm{~K}$, results in a $j_{c}$ of $3 \times 10^{9} \mathrm{~A} / \mathrm{m}^{2}$. Such large values of $j_{c}$ and $d$ yield a rather smaller Josephson penetration depth $\lambda_{J} \simeq 440 \mathrm{~nm}$, indicating that the weak link normalized length is $\ell=w / \lambda_{J} \simeq 2$. The threshold current $I_{c}(B)$ for a long overlap-type Josephson junction with uniform bias and asymmetric boundary conditions was numerically computed for $\ell=8$ and is shown in Fig. 4.
For qualitative modelling, we assumed a uniform current distribution, which leads to a larger normalized length than inferred from the experiment. In the real junction, the bias current flow along the $\mathrm{Nb}$ edges ${ }^{11,12}$ which makes the effective current density larger, the real Josephson penetration depth shorter and the true junction normalized length larger, as suggested by the numerical simulations.

In summary, we measured and qualitatively explained the behaviour of the planar Josephson weak link with the ferromagnetic injectors. To explain Fresnel-like $\mathrm{I}_{c}(\mathrm{~B})$ pattern, we employed the model of Josephson junction exposed to magnetic field gradient. Via current injection from the ferromagnet into the superconductor, we could control the amplitude of critical current and the period of $\mathrm{I}_{c}(\mathrm{~B})$ dependence, i.e., area of the junction. For quantitative explanation, one has to take into account the non-uniformity of the current flow via $\mathrm{Nb}$ strip. The impact of spin-polarization on the properties of such junctions can be investigated by the use of different ferromagnetic materials as injector and their additional polarization by in plane magnetic field.

We thank H. Kohlstedt, Š. Gaži, I. Vávra, and R. Soni for fruitful discussions. Authors wish to acknowledge the SFB 689 project and DFG via KO 1953/11-1.

${ }^{1}$ B. D. Josephson, Phys. Lett. 1, 251 (1962).

${ }^{2}$ J. Pearl, Appl. Phys. Lett. 5, 65 (1964).

${ }^{3}$ A. F. Morpurgo, T. M. Klapwijk, and B. J. van Wees, Appl. Phys. Lett. 72, 966 (1998).

${ }^{4}$ J. J. A. Baselmans, A. F. Morpurgo, B. J. van Wees, and T. M. Klapwijk, Nature 397, 43 (1999); Superlattices Microstruct. 25, 973 (1999).

${ }^{5}$ J. Huang, F. Pierre, T.T. Heikkila, F. K. Wilhelm, and N. O. Birge, Phys. Rev. B 66, 020507 (2002).

${ }^{6}$ O. Vávra, W. Pfaff, and Ch. Strunk, Appl. Phys. Lett. 95, 062501 (2009).

${ }^{7}$ M. A. Sillanpaa, T. T. Heikkila, R. K. Lindell, and P. J. Hakonen, Europhys. Lett. 56, 590 (2001).

${ }^{8}$ R. Monaco, J. Appl. Phys. 108, 033906 (2010).

${ }^{9}$ R. Monaco, J. Mygind, and V. Koshelets, Appl. Phys. Lett. 98, 072503 (2011).

${ }^{10}$ Given the symmetric geometrical configuration of the samples, the self induced field has to be ascribed to some asymmetry in the chip wiring.

${ }^{11} \mathrm{~A}$. Barone and G. Paterno, Physics and Applications of the Josephson Effect (Wiley, New York, 1982).

${ }^{12}$ M. R. Samuelsen and S. A. Vasenko, J. Appl. Phys. 57, 110 (1985). 